\title{
FRAMEWORK FOR COUPLED DIGITAL TWINS IN DIGITAL MACHINING
}

\author{
D. Plakhotnik ${ }^{1,2 *}$, A. Curutiu ${ }^{2}$, ,A. Zhulavskyi ${ }^{3}$, Xavier Beudaert ${ }^{4}$, Jokin Munoa ${ }^{4}$, M. Stautner ${ }^{1}$ \\ ${ }^{1}$ ModuleWorks GmbH, Aachen, Germany \\ ${ }^{2}$ Module Works SRL, Bucharest, Romania \\ ${ }^{3}$ Sumy State University, Sumy, Ukraine \\ ${ }^{4}$ IDEKO S Coop: Elgoibar, Spain
}

*Corresponding author; e-mail: denys@moduleworks.com

\begin{abstract}
This paper presents a further elaboration of the use of the digital twin concept in digital machining. The main goal of the research is an attempt to combine digital representations of different objects involved in the machining process in a holistic manner. Digital twins of the in-process workpiece, cutting tool, clamping, and machine tool along with the process twin are connected in a framework in which all these elements can influence each other. The framework shows different levels of integration of different digital twins and practical recommendation on the implementation. Besides, the framework supports a simulation layer that provides data for the intensity of the interaction. Such interactions can result in, for instance, cascading calculations of cutter workpiece engagement, cutting forces, tool wear, tool deflection, chatter, and so on. Eventually, a design for a software mockup was elaborated to present the developed framework. The entire workflow to simulate part machining in such a digital representation can be used as an ultimate tool for CAM simulation and NC verification within the Zero Defect Manufacturing paradigm.
\end{abstract}

\section{Keywords:}

CAM; digital twin; digital machining; NC verification

\section{INTRODUCTION}

The concept of the Digital Twins gets more and more traction in the manufacturing industry. Many scientific publications address this topic and try to elaborate on further bits of how to use digital twins. The number of relevant papers reached a significant amount. Recent survey papers ([Lechler 2020] and [Sjarov 2020]) report that there exist at least 40 studies about digital twins in manufacturing. Many researches see digital twins from different perspectives and apply digital twins in different applications. This leads to uncoherent understanding and multiple definitions of digital twins.

The general idea is to represent a physical object, also often called 'physical asset', in a digital format. In the manufacturing world, this idea was in the air because of the intensifying use of software tools at every step, as shown in Fig. 1. The very beginning of the production chain has already been digital. Parts are designed in CAD software, their digital copies can be stored with all revisions throughout the entire lifecycle. Then, the CAD part is used for CAM planning. Toolpath generation and CAM simulation utilize a completely digital approach to create a digital copy of the part after machining. In the meanwhile, the cutting tools are also represented in the digital manner. The next step introduces some traits from the real world. In order to generate NC code, CAM programs must be provided with the information about machine tool kinematics and specifications of the commands compatible with the CNC unit. Ultimately, the last step happens in the real machine, where the part is fabricated.

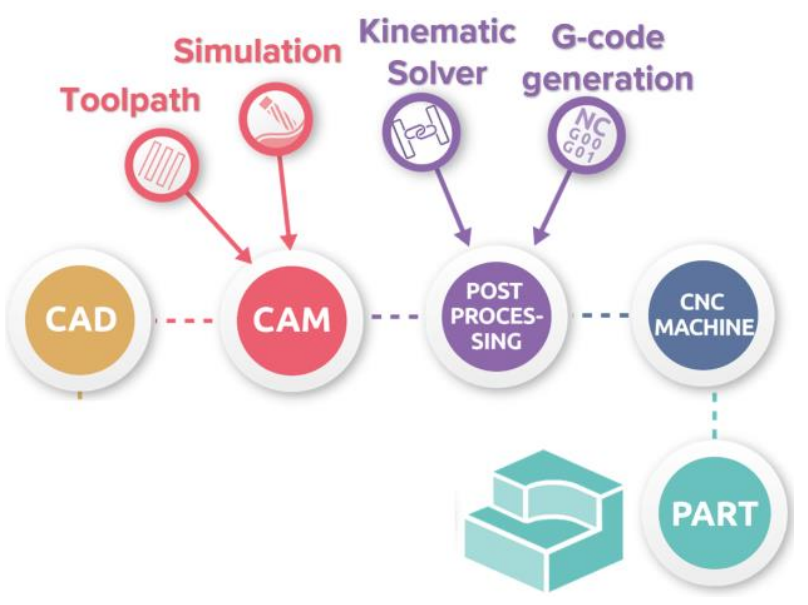

Fig. 1: Classical production workflow. 
The actual machining process involves all physical actors and results into the actual part. All machined parts are unique. No one is identical to another because there are always variations in the material properties, process parameters, etc. These differences cannot be foreseen by the classical CAM simulation, which is often believed to be a kind of a digital twin. Relying on CAM simulation contradicts, to a large extent, the concept of representing unique physical assets, because CAM simulation inherits no uncertainties that come from the real world making each part unique. Therefore, the crucial role in the digital twin concept should play so called "twinning" [Jones 2020]. Twinning is about synchronizing the virtual and physical states through updating virtual parameters with the actual physical parameters, as shown in Fig. 2. Since the simulation models underlying the digital twin are not complete and do not represent the real world exactly, the digital twin tends to deviate more as time passes. Therefore, the twinning should be scheduled often enough to keep the digital twin close to its physical origin.

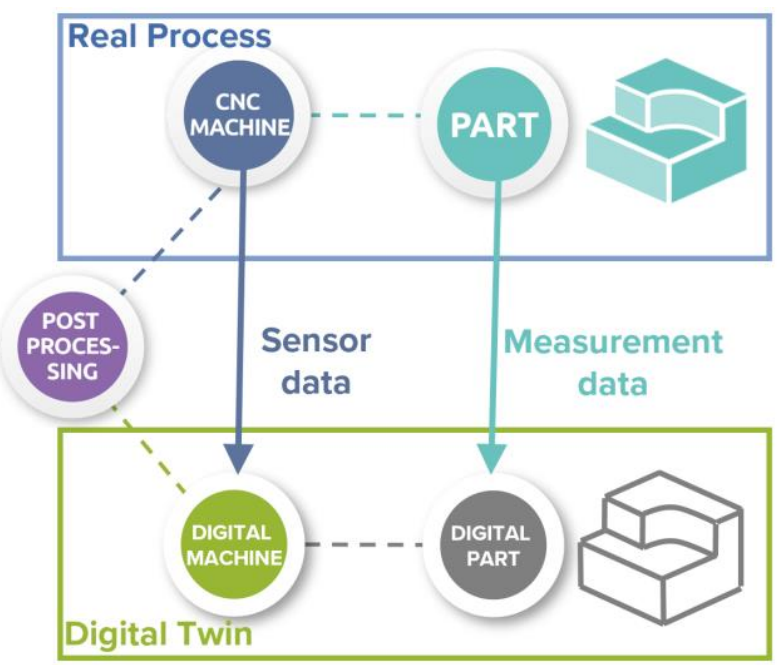

Fig. 2: Synchronizing the digital and real environments.

Although machining involves various mechanical systems, in the context of the digital twin, different systems are evaluated rather independently [Schleich 2019]. Depending on the focus of the research or practical interests, the core element of the digital twin can be either a cutting tool or machine. The part or in-process workpiece has an interesting role. Usually, during the machining process, the stock material undergoes very significant shape transformation - a lot of material are being removed by cutting tools. Twinning of the part is not feasible at decent rate because measurement requires pauses in the process, whilst almost not bringing any tangible added value. Twinning of the part is usually possible only at the end of the fabrication or between operation. Therefore, the digital twin of the part depends heavily on CAM simulation. CAM simulation is the core component that may bind together all digital twins involved.

Inevitable, the cutting tool is very important in the machining process. The cutting tool operates in extreme conditions that change the tool shape. With the knowledge about the tool, monitoring and predicting tool failure became the key goals of the digital twins of cutting tools. [Botkina 2018] elaborated a data format and data management for a digital twin of the cutting tool to trace data about the tool's ongoing machining operation, toolpath, forces and torques, time of usage, etc. [Kannan 2018] elaborated a digital twin specialized for grinding wheels to store process relevant information. In addition to storing historical data, some digital twins also included a simulation model to predict tool wear ([Christiand 2020], [Polini 2020], and [Lynn 2018]).

The machine tool is another major element of the machining process. Like cutting tools, the machine tool dictates the final outcome of the part quality. Since the machine motions do not ideally coincide with the programmed path, the actual cutting tool path is distorted and introduces geometrical deviations. Monitoring-based simulation can acquire real values of machine axes and use them in CAM simulation. This approach results in a more genuine NC verification ([Armendia 2019], [Plakhotnik 2019], and [Zhao 2019]). Sometimes, the machine can be modelled in a more sophisticated way to include FEM simulation predicting structural behavior of the machine [Scaglioni 2018].

The cutting process can become another subject of the digital twin as well. The transient parameters recorded during the cutting process can be used together with the deep learning approach to predict tool wear [Qiao 2019].

Weak coupling of digital twin modules in CAM systems does not allow efficient use of information available during the process. The goal of this paper is to propose a CAMbased framework integrating several digital twins. The framework was devised to ease software implementation within existing CAM software.

\section{DATA SPECIFICATION}

The digital twin paradigm does imply no limitation on the detail level of the virtual representation. Basically, all kind of information describing the physical asset from nanoscale to macroscale are allowed. However, the consumption of computer memory remains a great concern. In the ideal case, the amount of data should be reduced to minimum that would allow restoring object's properties and geometry. For example, a milling tool can be described by a set of specifications, like a $2 \mathrm{D}$ rotational profile, number of flutes, flute profiles, etc. However, generalization using pure specifications and geometric dimensions may not be appropriate for digital twins because it prohibits customization or fine tuning of the digital twin to meet specific features of its physical twin. Specifications must be extended with additional data structures capable of comprehending extra data from the real world. Besides, some properties might not be measurable and be corresponding to intangibles, like the kinematic structure or definition of collision bodies, as shown in Fig. 3.

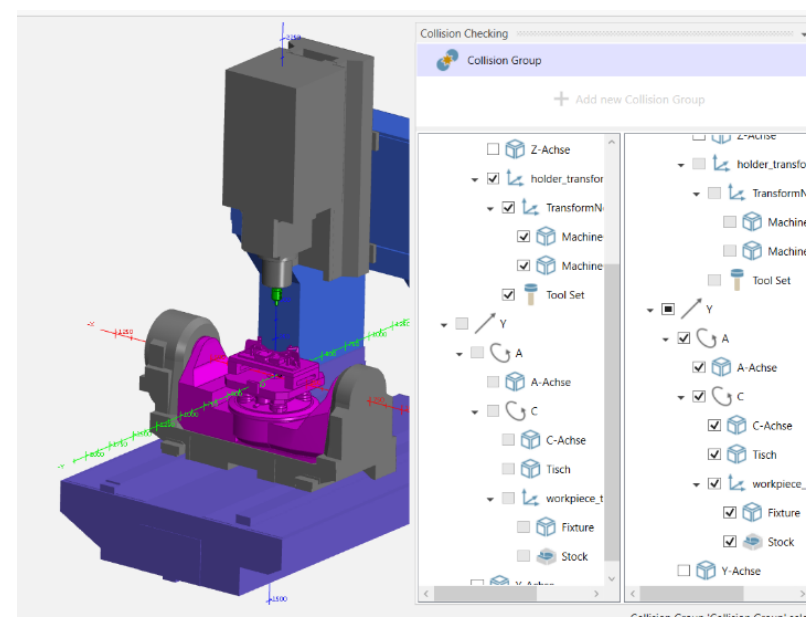

Fig. 3: Machine tool definition along with the CAD and kinematic structure.

Since real world objects belong to the three dimensional space, in most of the cases, digital technologies must rely 
on a discrete representation resulting in $\mathrm{O}\left(n^{3}\right)$ memory use. The memory utilization can be reduced to $\mathrm{O}\left(n^{2}\right)$ but the reduced representation can describe only $3 D$ surfaces. Parts fabricated by utilizing conventional machining are well aligned candidates for digitalization with the surface-based definition. In most of the cases, the properties of the stock material can be assumed to be isotropic and homogeneous. At least, the inner material properties can be assumed constant, whilst the surface material properties may vary and be eventually described with the $\mathrm{O}\left(n^{2}\right)$ order of memory complexity. Parts fabricated by using additive technologies may not suit well to the surface-based definitions, since additive parts tend to have internal structures, designed lattice structures or unintended - porosities or voids between layers. The burden to define additive parts with complex surfaces may eliminate benefits of $\mathrm{O}\left(n^{2}\right)$.

$\mathrm{O}\left(n^{3}\right)$ space complexity can still be used when $n$ is relative small. For instance, a digital twin of a machine tool may include a rough FEM model to compute natural frequencies. A good practice would be to combine surface and volumetric representations with different resolutions that fit a given purpose.

\subsection{Transient processes}

Another critical aspect is how to consider that the object states and processes are transient by their nature. The cutting tool deteriorates and wears; machine tools loose their accuracy, and so on. In many CAM applications it would be too expensive and time consuming to capture transient processes by twinning with the real world, especially in the case of the first part right approach.

The digital twin must include a simulation module that would allow sophisticated simulation and/or either interpolation or extrapolation of the system state during machining. As in simulation solvers, selection of the time-step is crucial - too much data will overwhelm the simulation system. Instead of a fixed time step, a variable time step provides more flexibility. Within one time step, parameters may be assumed to remain either constant or to change linearly. However, higher orders may still be needed, for instance, for simulation of CNC interpolation. Constant values can be added as properties of single tool moves, since the tool move is a fundamental object type in CAM simulation. Constant values are straightforward to visualize as 1D data (as in Fig. 4) or 2D data mapped on the machined surface (as in Fig. 5).

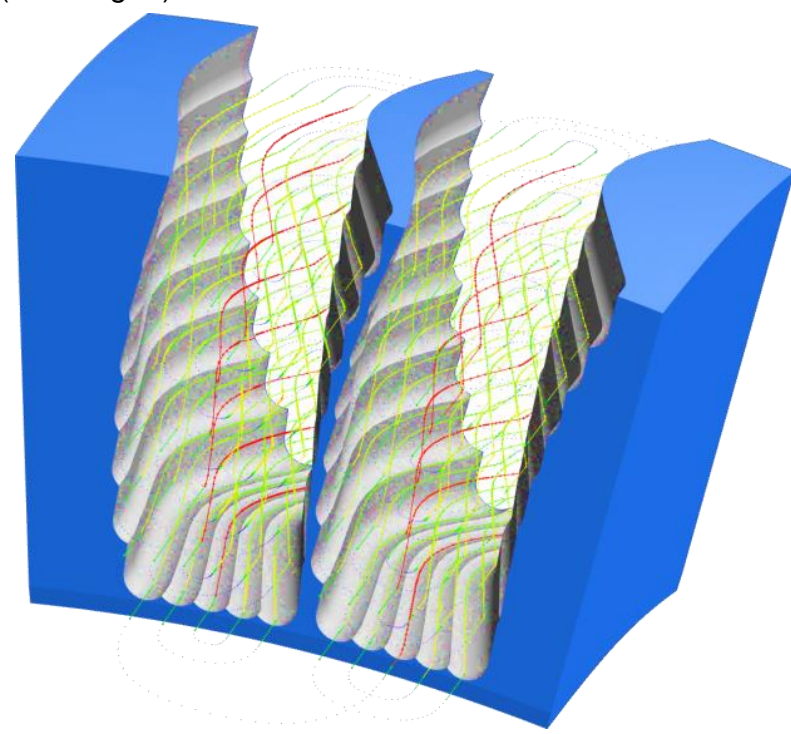

Fig. 4: Color coding of toolpath (backplot) with the material removal rate (green - less, red-more).

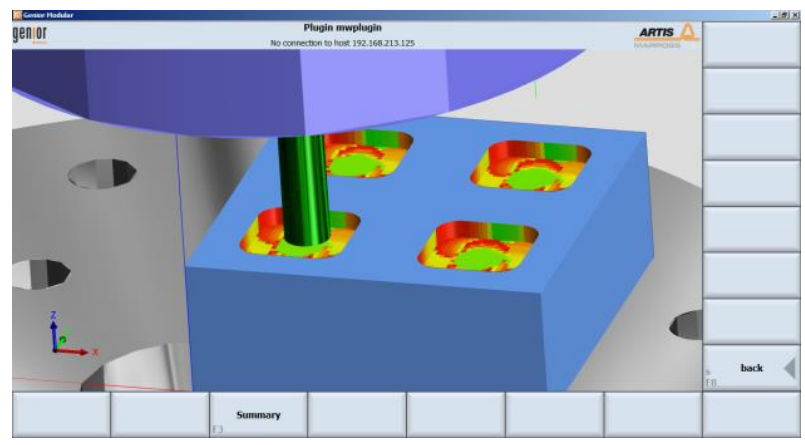

Fig. 5: Color coding of machined pockets with measured spindle torque (green - less, red-more).

Workpiece simulation is the central component of the digital twin because it predict the outcome of machining, including potential collisions between moving elements.

When the in-process workpiece changes overtime, a lot of information is literally removed with the chip material. Fig. 6 depicts how chatter influences the machined surface. At the end of track 1, tool chatter is more intense (red spot). However, on track 2, which is a longer version of track 2, chatter marks at the same position can not observed because the surface with chatter marks was removed. This case might be important for the cutting tool performance but, certainly, not for the final part. Nevertheless the cutting tool may change and influence the part but this tool shape change must be spotted by tool's digital twin and communicated back to CAM simulation.

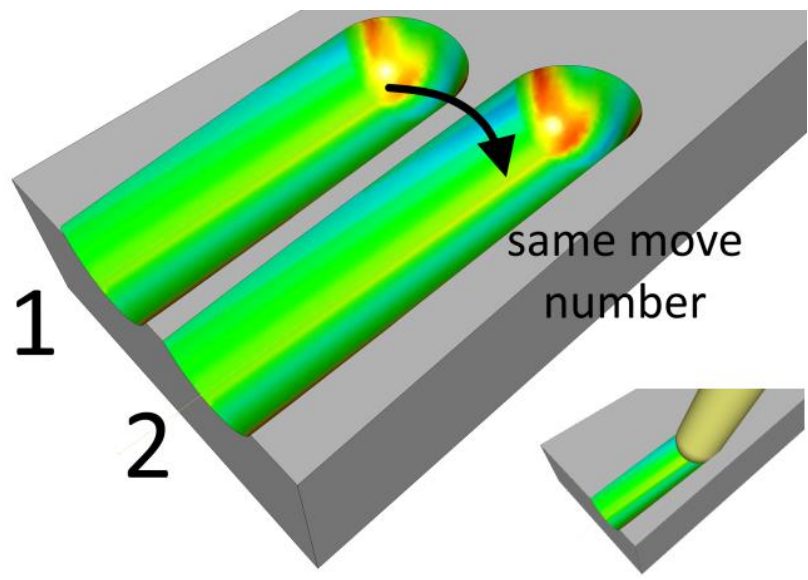

Fig. 6: Simulated chatter values (synthetic values coded from blue to red) in two slots with identical conditions.

\subsection{Data granularity/resolution}

Data granularity is about how detailed the simulation should be. Regardless whether there is $\mathrm{O}\left(n^{3}\right)$ or $\mathrm{O}\left(n^{2}\right)$, the absolute value of $n$ is important. Data granularity $n$ is about how detailed the simulation should be. If a single value $(n=1)$ is assigned, then, a property or any other parameter remains constant within the defined domain. For instance, a single value tool wear means that such a wear value is assumed at every point of the cutting edge. For geometry, it may mean the use of a simple curve or surface, e.g. a sphere defined by its radius. For a milling tool, it may mean a perfectly round ball end. Such single value parameters are well suited to simulation algorithms. Continuous domains (uniform surfaces, constant speeds, constant feed, etc.) provide enough room for optimization of computations routines. However, due to the purpose of digital twins, in which uniqueness of each physical part must be identified, single values are often out of the scope. 
The precision of the geometric model depends on the underlying data structure. Practically, commercial CAM systems use either mesh-based data structures (triangulated surfaces) or multi-dexel models. Each approach has its advantages and drawbacks. However, the use of dexel data structures is more stable in terms of memory consumption and lower upper bound for the tool swept volume computation. Besides, multi-dexel models can be used to restore surface information, as shown in Fig. 7. The distance between dexels must be chosen such that it can describe the smallest geometric features. Loca refinement of simulated results can provide more fine details of the surface with some overhead costs of simulation on demand, as shown in Fig. 8.

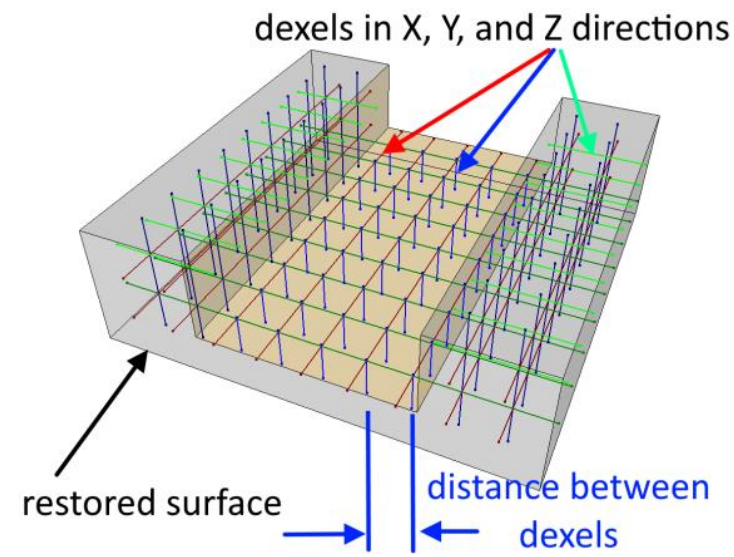

Fig. 7: Simulated chatter values (synthetic values coded from blue to red) in two slots with identical conditions.

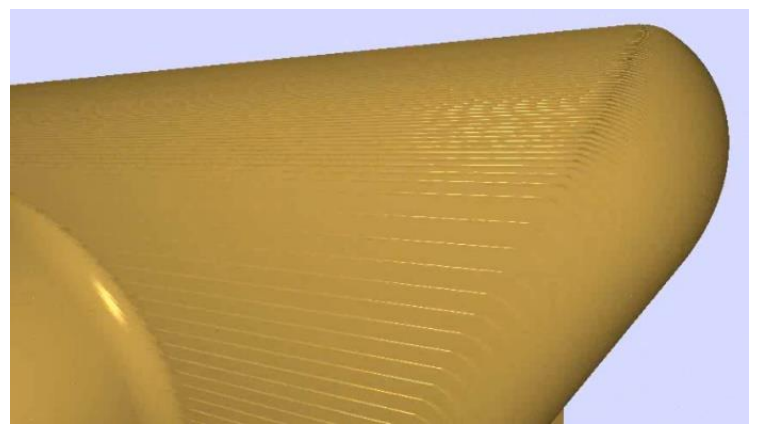

Fig. 8: Visualization of small scallop features after milling simulated on demand by shader rendering.

The surface of the tool surface may be represented not as a rotational-symmetric contour but as a pixels map, as shown in Fig. 9.

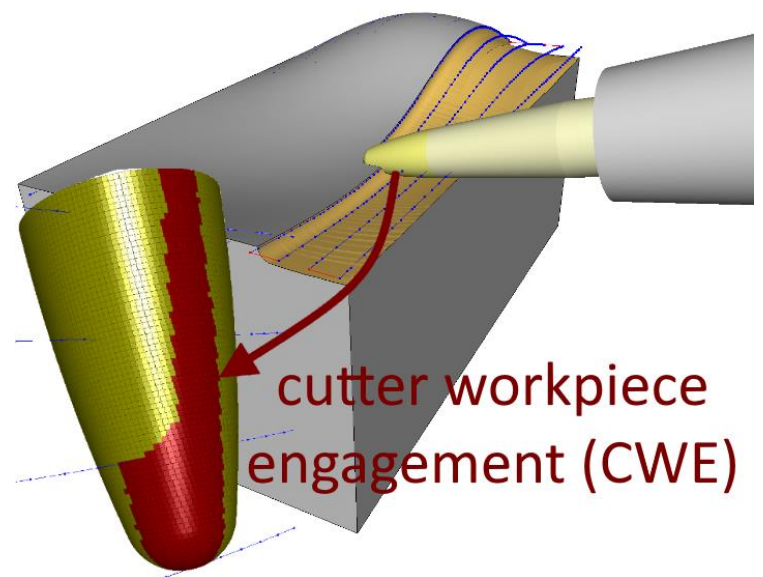

Fig. 9: Pixelized surface of the rotating tool to represent cutter workpiece engagement during milling.

\section{COUPLING}

Each pixel on the cutting tool may keep the history of all tool moves in which the pixel was in the cutter workpiece engagement (CWE), spindle speeds, feeds commanded, and the duration of the moves. Since this information belongs to the digital twin of the cutting tool, it may be further extended to store information about conditions that influence the tool life. That can include material properties of the workpiece, whilst one tool can be used for different parts which underwent, for instance, different thermal treatment. If the digital twin of the workpiece holds material property information, digital twins of the cutting tool and the workpiece can be coupled (connected) through a software interface that would communicate the material property from the workpiece's digital twin to the tool's digital twin. Accordingly, the machine tool's digital twin may share the state of coolant, spindle speed, actual feed, measured torques, etc.

Fig. 10 shows a screenshot from CAM simulation that takes synthetic data from tool motion and tool wear simulations. CAM simulation must break the tool move into smaller steps because CAM simulation cannot change tool geometry within one move. Therefore, each iteration has the following steps:

1. The digital twin of the cutting tool provides the CAM simulation with the actual tools profile (worn one).

2. The digital twin of the machine tool provide the CAM simulation with the tool move coordinates (based on simulated or measured data).

3. CAM simulation performs material removal simulation and communicates CWEs to the digital twin of the cutting tool.

4. The digital twin of the cutting tool requests process information from the digital twin of the machine tool, and updates tool's actual profile, which is computed in a tool wear simulation module inside the digital twin of the cutting tool.

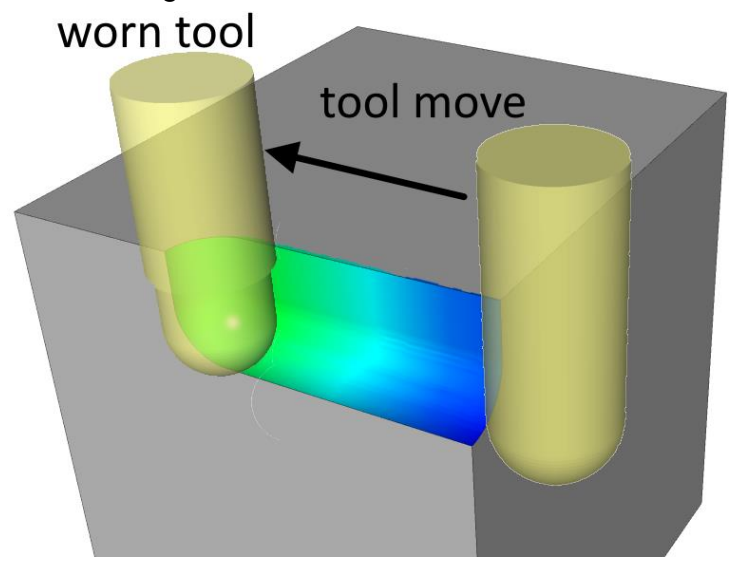

Fig. 10: A CAM simulation for mock-up data with a tool being worn during machining. The color from blue to green depicts the different tool wear values.

The concept can be elaborated to support more phenomena. The digital twin of the cutting tool may simulate cutting forces, chatter, and deformations. The digital twin of the machine tool may be extended to support simulation of thermal and structural behavior, collision detection, and other aspects that might be important for an implementation. Respectively, the digital twin of the workpiece can, for example, include an algorithm that would consider internal and induced stresses and, accordingly, part distortions caused by the internal stresses and cutting forces. Taking part deformations into account can be 
beneficial for machining of thin-walled structures. However, coupling of digital twins must be done cautiously. The suggested coupling assumes that the bodies involved (tool, workpiece, machine tool, fixture, etc.) are rather absolutely rigid, and they remain unchanged until the end of each iteration. If the bodies are flexible (like in 5-axis blade milling, in which both the blade and a long tool may deflect due to cutting forces), each iteration needs one more embedded simulation that finds an equilibrium state where blade deflection changes the cutting force, the changed cutting force changes deflections of the tool and the blade, an so on, until the computation converges.

Moreover, the results of CAM simulation must be available for virtual metrology to avoid measurement of the actual part, as shown Fig. 10.

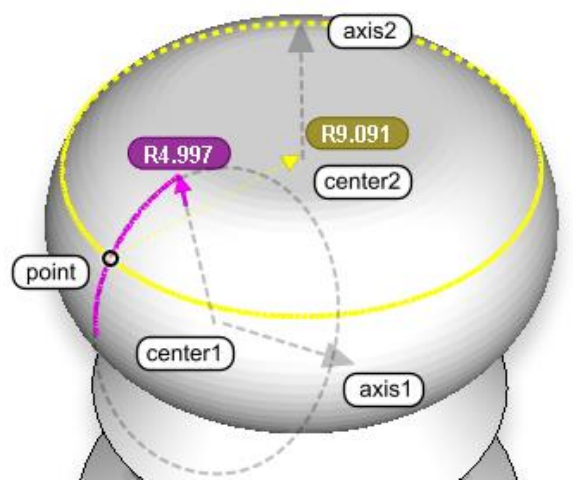

Fig. 10: Measurement of the machined part in CAM simulation.

The ultimate goal of a digital twin based on CAM simulation could be not only the prediction of the machining operations, but also optimization of the process. The suggested approach can be embedded into toolpath generation software to compensate the tool wear. Fig. 11 illustrates an example of how the toolpath can be modified to compensate the change of the tool size due to wear.

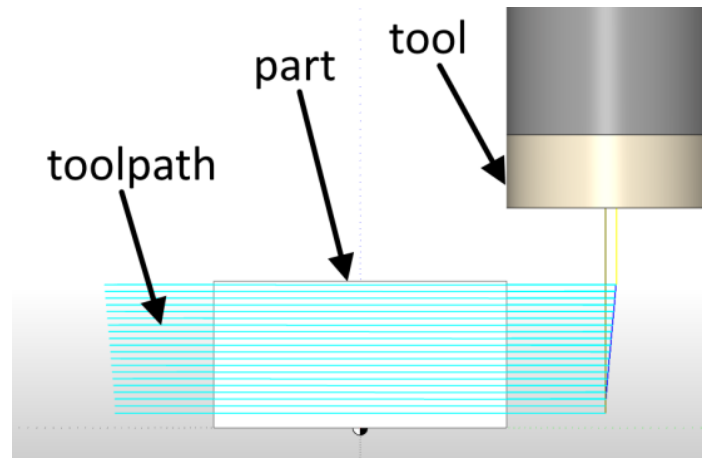

Fig. 12: Optimization of the toolpath based on compensation of the tool wear.

\section{SUMMARY}

This paper summarizes initial findings about algorithms and data structures that can be embedded or connected to commercial CAM software in order to accelerate the adoption of the digital twins in digital machining. The developed concepts are suitable for software implementation.

\section{ACKNOWLEDGMENTS}

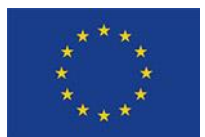

This research was partially funded from the European Union's Horizon 2020 Research and Innovation. Programme under the project InterQ (grant agreement No. 958357) and the project Twin-Control (grant agreement No. 680725) and it is an initiative of the Factories-of-theFuture (FoF) Public Private Partnership.

\section{REFERENCES}

[Armendia 2019] Armendia, M., et .al. Evaluation of Machine Tool Digital Twin for machining operations in industrial environment. Procedia CIRP. 2019, 82, 231-236

[Botkina 2018] Botkina, D et .al. Digital Twin of a Cutting Tool. Procedia CIRP. 2018, 72, 215-218

[Christiand 2020] Christiand and Kiswanto, G. Digital Twin Approach for Tool Wear Monitoring of Micro-Milling. Procedia CIRP. 2020, 93, 1532-1537

[Jones 2020] Jones, D., et .al. Characterising the Digital Twin: A systematic literature review. CIRP Journal of Manufacturing Science and Technology. May 2020, 29 , 36-52

[Kannan 2018] Kannan, K. and Arunachalam, N. A Digital Twin for Grinding Wheel: An Information Sharing Platform for Sustainable Grinding Process. Journal of Manufacturing Science and Engineering. December 2018, 141, 2

[Lechler 2020] Lechler, et .al. Introduction of a comprehensive Structure Model for the Digital Twin in Manufacturing, 2020 25th IEEE International Conference on Emerging Technologies and Factory Automation (ETFA), 2020, pp. 1773-1780

[Lynn 2018] Lynn, R., et .al. "Realization of the 5-Axis Machine Tool Digital Twin Using Direct Servo Control from CAM" in NIST Model Based Enterprise (MBE) Summit 2018, April 2018, Washington, D.C

[Plakhotnik 2019] Plakhotnik, D., et .al. Visualization of Simulated and Measured Process Data. 2019, TwinControl. Springer International Publishing. 225-233. ISBN: 978-3-030-02203-7

[Polini 2020] Polini, W. and Corrado, A. Digital twin of stone sawing processes. The International Journal of Advanced Manufacturing Technology, November 2020, 112, 1-2, $121-131$

[Qiao 2019] Qiao, et .al. Digital Twin for Machining Tool Condition Prediction. Procedia CIRP, 2019, 81, 1388-1393

[Scaglioni 2018] Scaglioni, B. and Ferretti, G. Towards digital twins through object-oriented modelling: a machine tool case study. IFAC-PapersOnLine, 2018, 51, 2, 613-618

[Schleich 2019] Schleich, B., et .al. Shifting value stream patterns along the product lifecycle with digital twins. Procedia CIRP, 2019, Volume 86, Pages 3-11, ISSN 22128271

[Sjarov 2020] Sjarov, et .al. The Digital Twin Concept in Industry - A Review and Systematization, 2020 25th IEEE International Conference on Emerging Technologies and Factory Automation (ETFA), 2020, pp. 1789-1796

[Zhao 2019] Zhao, G., et .al. Digital Twin for NC Machining Using Complete Process Information Expressed by STEPNC Standard. Proceedings of the 2019 4th International Conference on Automation, Control and Robotics Engineering, July 2019 